\title{
Recommender Systems: A Healthy Obsession
}

\author{
Barry Smyth \\ Insight Centre for Data Analytics, \\ University College Dublin, \\ Dublin, Ireland \\ barry.smyth@ucd.ie
}

\begin{abstract}
We propose endurance sports as a rich and novel domain for recommender systems and machine learning research. As sports like marathon running, triathlons, and mountain biking become more and more popular among recreational athletes, there exists a growing opportunity to develop solutions to a number of interesting prediction, classification, and recommendation challenges, to better support the complex training and competition needs of athletes. Such solutions have the potential to improve the health and well-being of large populations of users, by promoting and optimising exercise as part of a productive and healthy lifestyle.
\end{abstract}

\section{Introduction}

Recommender systems learn about the preferences and needs of users in order to deliver more personalised online experiences. Techniques such as collaborative filtering and content-based recommendation harness ratings, metadata, and other forms of content, to make suggestions to users based on their stated, or inferred, likes and dislikes; see (Ricci, Rokach, and Shapira 2015; Bridge et al. 2005; Smyth 2007; Burke 2002). Such techniques have proven to be particularly successful in many e-commerce and entertainment settings, so much so that today recommender systems are increasingly influencing the books we read, the music we listen to, and the movies we watch.

We can usefully view the above as the first generation of recommender systems. As for the next generation, the central message in this paper is that this will see the emergence of a new type of recommender system, one that will reach beyond the online world of movies and music, and into the 'real-world' of how we work, rest, and play. In particular, we highlight an important opportunity when it comes to helping people to live healthier lives. Of course there is a long history of $\mathrm{AI}$ and machine learning in classical medicine and healthcare (Peek et al. 2015), but in this work we focus instead on ways to promote and support physical activity and exercise, as a vital part of staying healthy well into old age. This is motivated by a coincidence of factors:

1. The ubiquity of always-on, fully-connected, locationaware, mobile devices means that many aspects of our

Copyright (c) 2019, Association for the Advancement of Artificial Intelligence (www.aaai.org). All rights reserved. everyday lives can be recorded and analysed to better understand our activity patterns. This provides a near constant supply of rich data about our habits and routines.

2. It is an inconventient truth about human evolution that our bodies are adapted for the type of physical activity that is associated with hunter-gatherer lifestyles, rather than the sedentary habits enabled by modern society. This is taking its toll in the developed world, with the emergence of common disease states (obesity, diabetes, osteoporisis etc.) that appear to arise, in large part, because our bodies are maladapted for modern society.

3. As people are becoming better informed about the importance of an active lifestyle, they are seeking out new ways to be more active, become fitter, and stay healthy, often looking to mobile apps as key tools in this quest.

With this in mind, we will present the case for recommender systems as a way to nudge people towards healthier lifestyles. We will argue that they are particularily well suited to popular endurance sports, while calling out a variety of interesting and novel recommendation tasks that will be familiar to many runners, cyclists, and triatheletes.

\section{Escaping the Exercise Trap}

In our always-on, connected world almost everything we do generates a data record that is stored somewhere (Campbell et al. 2008). While this brings its own set of issues, especially when it comes to safe-guarding the privacy rights of individuals, it also affords new opportunities when it comes to better understanding, at scale, how people live, work, and play (Mayer-Schönberger and Cukier 2013; Rooksby et al. 2014). And this has important implications for modern society, from transport and energy policy, to healthcare and security, and many more besides.

\section{Small Sensors \& Big Data}

Recently, there has been an explosion in the number of apps, sensors, and devices that are concerned with collecting, analyising, and communicating our personal health and activity data. Indeed it is now unusual to see people exercising without some evidence that they are recording their activities, whether via a mobile phone strapped to their arm, the latest 
GPS-enabled, heartrate-sensing smartwatch, or a new wearable sensor to analyse their gait, effort, or posture. Manufacturers like Garmin, Fitbit, and Polar have forever changed the way that many of us exercise, by counting our steps, tracking our routes, weighing our efforts, and measuring our sleep patterns.

The personal data that results can bring valuable insights to bear on longitudinal health studies, but only if we can develop the tools to discover and understand the patterns within. This is where AI and machine learning has the potential to radically change how we live our lives (MayerSchönberger and Cukier 2013), and may prove to be transformative for modern societies, as they struggle to cope with increasingly aging populations, and the strain that they place on conventional healthcare infrastructure. It is an unfortunate reality that most healthcare systems prioritise caring for the ill rather than helping us to remain well, to avoid falling ill, in the first place.

\section{Exercise as the Best Medicine}

We all know that exercise is generally good for us, and that we probably do not get enough of it, but the reasons for this are revealing as we consider how to harness technology to promote healthy lifestyles among the wider population (Möller et al. 2011; Yoganathan and Kajanan 2013).

One explanation as to why exercise is so positive is that, quite simply, we are born to run (Mattson 2012; Bramble and Lieberman 2004). As early hominids ventured forth from the cover of the forest canopy, and into the Savannah grasslands of ancient Africa, they transitioned from a quadrupedal gate to a bipedal one. As a consequence of this they gave up the ability to run fast, but they gained the ability to run far, because evolution discovered new ways to keep hominids cool under the heat-stress of prolonged exercise. For example, sweating to keep cool is a key feature that distinguishes hairless humans from many other animals.

It is certainly true that hunter-gatherers were no match for the strength or speed of big-game quadrapeds, but quadrapeds must pant to stay cool, and panting is incompatible with the galloping gate that gives quadrapeds their speed advantage. This means that quadrapeds cannot sustain their speed over long distances. Instead they must slow to a trot in order to cool-down. However, the gallop-trot transition speed was slower than the running speed of early humans, which gave them an distinct advantage over long distances. Consequently, early humans hunted using a persistence hunting strategy, tracking prey to heat exhaustion over very long distances (Liebenberg 2008).

One important consequence of this is that modern humans are adapted for regular amounts of endurance activity well into late age. Indeed the field of evolutionary medicine is devoted to better understanding the consequences of the these past selection pressures when it comes to our health in modern environments. So-called mismatch diseases - such as, obesity, type-2 diabetes, osteoporosis, and depression have been proposed as the consequences of our maladapted hunter-gatherer genes (Dearden 2007). These diseases are otherwise rare, largely preventable, and almost entirely absent from well-adapted, hunter-gatherer populations. It is a paradox of the modern world that reduced mortality comes with a significant increase in morbidity, as we suffer from these chronic, largely preventable diseases for long periods at the end of our lives.

The evidence suggests that these mismatch diseases are a consequence of physical inactivity. Conversely, studies have also demonstrated the benefits of physical activity, with significant improvements in all-cause mortality across active populations relative to inactive ones (Lee and Skerrett 2001).

\section{The Exercise Paradox}

The message seems to be that exercise is not just good for us, it is ultimately necessary for good health. In a very real sense exercise can be the best medicine when it comes to many mismatch conditions: it comes with much lower economic costs and far fewer side-effects than conventional therapies. And, as our society trends older, regular exercise can make a real difference when it comes to our quality of life well into old-age. But if evolution has created our bodies to be exercise machines, which require regular physical activity in order to stay healthy, then why is modern life distinguished by a lack of physical activity? Why aren't we craving the activity levels that our bodies appear to need to stay healthy? And why are we so willing to live sedentary lives?

The cruel joke that evolution has played is that, while on the one hand we are adapted for physical activity, on the other hand our hunter-gatherer genes also prioritise rest and inactivity, because they were selected for in an environment where food and energy were limited resources. This is the exercise paradox: on the one hand we need to exercise, but, on the other, we can be easily tricked into avoiding it, to conserve the energy that our bodies believe is still in scare supply, which it is not.

Thus, we need to work hard to overcome our sedentary proclivities and this is where, we argue, technology can play an important role, by making it easier and more appealing for people to increase their activity levels; see for example, (Hermens et al. 2014). We need to develop technologies that can nudge us towards healthier choices by recognising our routines and understanding our habits, and by using persuasive recommendations to transform unhealthy behaviours into more healthy habits. In the remainder of this paper we will focus on one practical example of how we might help people to exercise more effectively, by assisting recreational endurance athletes to train for, and compete in, challenging events such as marathons and triathlons.

\section{Endurance Exercise as a Novel Recommender Systems Domain}

As people become increasingly aware of the importance and value of regular exercise in their lives, more and more are turning to popular endurance sports to challenge themselves. Every year millions of people participate in running, cycling, swimming, and triathlon events, many for the first time. Such events, and the training activities that go with them, provide a fertile set of recommender system challenges and novel applications, for a variety of reasons: 
1. There exists a large base of highly motivated, yet often inexperienced users, who are actively seeking out advice and guidance on many aspects of their chosen sport, from training and injury prevention advice, to equipment recommendations and race planning.

2. These users generate a plentiful supply of detailed data (training activities, rest/recovery, nutrition, race results etc.) which can be used to better understand their abilities, preferences, and goals.

3. Training for, competing in, and recovering from, endurance events, such as the marathon or triathlon, encompasses a variety of different recommendation tasks and opportunities, as we shall discuss, and the ubiquity of mobile devices presents a unique opportunity to offer users real-time solutions to these challenges.

In the remaining sections we will sample a number of these recommendation tasks and opportunities, linking them to existing research where feasible.

\section{Fitness Estimation \& Training Effects}

Sports scientists use a variety of important laboratory metrics to estimate the fitness levels of individuals and how they change under training conditions. The well-known $\mathrm{VO}_{2} \max$ score measures the maximum rate of oxygen consumption during exercise. It reflects the cardiorespiratory fitness of an individual and is an important determinant of their endurance capacity during prolonged exercise. But it is usually measured in a laboratory setting and, as such, not necessarily accessible to recreational athletes. With the advent of smartwatches and wearable heartrate sensors it is possible to estimate $V 0_{2}$ max based on training effort under specific conditions. For instance, there are a number of examples of recent research on the use of machine learning for $\mathrm{VO}_{2} \max$ prediction (Akay et al. 2011; Abut, Akay, and George 2016) but many challenges remain to improve prediction accuracy under real-world, recreational training conditions. These have the potential to provide accurate $V 0_{2} \max$ estimates without the need for expensive laboratory support.

Similar approaches can to be applied to predict a wide range of other key performance metrics, such as a runner's lactate threshold ${ }^{1}$, or the training effect of a specific session, which is a measure of fitness improvement. These estimation problems can be readily framed as classical supervised learning tasks and the resulting models may transform the effectiveness of training programmes by providing personalised advice and tailored recommendations about how an athlete should train on a given day in terms of their target pace, duration, and effort.

\section{Training Session Classification}

When it coms to training, runners and cyclists talk in terms of intervals, hill-repeats, tempo sessions, threshold training, fartleks, easy-days, progressions, ladders, speed-work,

\footnotetext{
${ }^{1}$ Lactate threshold refers to the pace at which the body can no longer effectively clear lactic acid from the muscles, which is produced as a by-product of exercise. For an athlete, this means a significant increase in discomfort and it will quickly lead to the need to slow-down significantly.
}

yasso-800s, etc. These are all different types of training sessions, designed to promote specific training effects. For example, running interval sessions, where the runner alternates between periods of fast running (for $400 \mathrm{~m}, 800 \mathrm{~m}$ or $1,500 \mathrm{~m}$ distances) and recovery, can improve aerobic and anaerobic endurance, increase $\mathrm{VO}_{2} \max$, and improve overall performance, while the increased 'afterburn' - referring to postexercise calorie consumption - can aid in weight-loss.

While training programmes prescribe a variety of different session-types, current apps do little when it comes to monitoring or assessing an athlete's adherence to specific training sessions. Most apps simply do not have any understanding of the nature or purpose of such sessions. They record GPS, pacing, and heartrate traces without encoding any of the key features that distinguish different sessions. This makes for a significant opportunity to draw on recent work about detecting structures and motifs in time-series (Senin et al. 2018; Berlin and Laerhoven 2012; Cheng 2013) in order to: (a) automatically classify training sessions to better assess a runner's performance or fitness level and, in due course, to adapt their training programme appropriately; and (b) to dynamically assess how well an individual is adhering to a particular training session, to provide in-session feedback (increase/decrease interval pace, reduce/extend interval duration, adjust recovery period etc.) and guide the individual to a better session outcome.

The ability to classify a training session, combined with accurate models of fitness and training effort, will make it possible to provide an individual with more targeted advice about the effectiveness of their training as well as pinpointing areas for improvement. This will help a runner understand whether they have pushed themselves too hard, or not hard enough, for instance, and can provide the basis for adaptations to a training programme to better balance activity and recovery.

\section{Recovery Prediction \& Training Load Estimation}

Indeed, recovery is a critical, but often overlooked, part of any training programme. Recovery allows the body to adapt to training and to replenish vital resources. Insufficient recovery can lead to missing out on fitness gains, and keeping track of recovery levels can reveal when training hard is likely to be beneficial or injurious to an athlete. An important opportunity exists to predict recovery needs, based on an athlete's current fitness levels, recent training effort, and key physiological indicators such as resting heartrate. While some fitness devices do include some recovery prediction features, they tend to be simplistic and offer considerable room for improvement. In the future, athletes will benefit from more insightful and actionable recovery recommendations, not only about how long they should recover for, but also about how they should recover and the type of activities they should and should not do (Glaros et al. 2003).

A related issue is training load, which provides a bigpicture estimate of an athlete's current training effort, and can be an important indicator of common problems such as over-training. Activity data provides a rich source of training data for machine learning, by integrating fitness and physiology data with training volumes, and user-provided train- 
ing assessments; e.g. by logging effort perceptions, documenting injury and illness. In due course, it may be possible to identify novel patterns linking fitness, training, recovery and injury and so develop effective early-warning systems for athletes, to alert them to changes in their performance, which may be a precursor to the onset of illness of injury.

\section{Personalised Training Programmes}

Perhaps the holy grail of recreational endurance athletes is the desire for personalised training programmes that are tailored for the precise needs and preferences of an individual. Most recreational athletes train using some form of training programme, usually one that they have found online, or one that they have adapted to their own needs over the years. These programmes will typically break a 12-16 week training period into a number of 3-4 week blocks, with each block made up of a number of specific training sessions in order to produce a given training effect (e.g. strength, endurance, etc.). Programmes may also include specific rest and dietary components.

It can be a challenge for an individual to find a training programme that suits their personal circumstances and goals, and many are left struggling to follow a mismatched, one-size-fits-all programme. Recently the concept of an virtual coach, capable of offering more personalised training advice, has been proposed in the literature (Fister et al. 2015; Rauter 2018), for resistance training and mountain biking. Similar ideas may be suitable to develop personalised programmes for endurance athletes, by harnessing accurate, real-time, personal measures of a individual's fitness, physiological well-being, training load etc. Personalised training plans can be generated by matching particular training needs with specific training sessions to provide the individual with specific guidance about how to conduct these sessions in terms of pace, effort etc.

How an individual responds to a given session, or training block, can be used to fine-tune future sessions or re-plan as needed. And finally, such personalised training recommendations can be augmented with supporting explanations so that the athlete can better understand the reason why a specific session is being recommended, how they should approach it, and how they should recover afterwards to gain maximum benefit.

\section{Goal-Time Prediction \& Pacing Planning}

So far we have focused on supporting individuals during training, but of course all of this training will be in the service of a specific event, or set of events, such as an upcoming marathon or triathlon. As an event approaches, an individual will start to plan their goal-time and race strategy. In endurance events, such as the marathon or triathlon, it is not enough to aim for a specific goal-time, it is just as important for participants to plan how to pace their race on the day, including their in-race nutrition to fuel their efforts, strategies to avoid hitting the wall, etc. (Smyth 2018).

When it comes to goal-time prediction there is a body of work that uses linear models to predict future race-times based on previous race-times; e.g. (Bartolucci and Murphy ). What is less well developed, however, is the translation of a goal-time into a specific race strategy and a concrete set of pacing recommendations. We have recently addressed this dual problem of goal-time prediction and pacing recommendation for marathons, by using case-based reasoning (Smyth and Cunningham 2017; 2018b; 2018a). In short, the goal-time and pacing plan for a target runner is adapted from the race-times and pacing profiles of runners with similar race histories. Recent research (Smyth and Cunningham 2018a) has considered different representations to encode the marathon history of runners and their impact on goaltime prediction accuracy and pacing-plan quality, demonstrating that accurate predictions, and high-quality pacingplans, can be generated for all levels of runner ability. The same approach can be readily adapted for other forms of endurance sport, and has the potential to offer valuable advice and in-race guidance to an individual as they plan for and compete in their chosen event.

\section{Conclusions}

We have proposed endurance sports as an interesting and challenging application domain for recommender systems and machine learning research. This is motivated by the importance of exercise to the human condition, a growing interest in endurance sports, and the plentiful supply of activity data from wearable sensors and mobile devices. The effort that needs to be invested by individuals as they train for, and compete in, endurance events reflects a complex mix of decisions and constraints that need to be carefully considered if participants are to remain healthy and effective during training and competition.

We have highlighted just some areas that are ripe for research, from fitness prediction to injury prevention, and from personalised training to effective race planning. There are others areas too that we have not mentioned, such as using machine vision techniques to analyse movement in order to analyse athlete form (gait, posture etc.) (Begg and Kamruzzaman 2005). We have cited recent research to highlight current efforts to address several of these challenges. By attempting to bring together and unify this early work, as part of a broader vision for a new generation of smarter training apps and virtual coaches, we hope that we can help to consolidate and catalyse future research in this exciting domain.

Our focus, on one end of the exercise-spectrum (endurance events such as the marathon) will not be for everyone. But the ideas presented can translate to other forms of exercise and activities at all levels, from $5 \mathrm{k}$ parkruns to casual walkers and Sunday cyclists, where helping people to get started, and then keep going, can make all the difference.

\section{Acknowledgments}

Supported by Science Foundation Ireland through the Insight Centre for Data Analytics under grant number $\mathrm{SFI} / 12 / \mathrm{RC} / 2289$

\section{References}

Abut, F.; Akay, M. F.; and George, J. 2016. Developing new vo2max prediction models from maximal, submaximal and questionnaire variables using support vector machines 
combined with feature selection. Computers in Biology and Medicine 79:182 - 192.

Akay, M. F.; Zayid, E. I. M.; Aktürk, E.; and George, J. D. 2011. Artificial neural network-based model for predicting VO2max from a submaximal exercise test. Expert Systems with Applications 38(3):2007-2010.

Bartolucci, F., and Murphy, T. B. A finite mixture latent trajectory model for modeling ultrarunners' behavior in a 24-hour race. Journal of Quantitative Analysis in Sports 11(4):193-203.

Begg, R., and Kamruzzaman, J. 2005. A machine learning approach for automated recognition of movement patterns using basic, kinetic and kinematic gait data. Journal of Biomechanics 38(3):401-408.

Berlin, E., and Laerhoven, K. V. 2012. Detecting leisure activities with dense motif discovery. In The 2012 ACM Conference on Ubiquitous Computing, Ubicomp '12, Pittsburgh, PA, USA, September 5-8, 2012, 250-259.

Bramble, D. M., and Lieberman, D. E. 2004. Endurance running and the evolution of homo. Nature 432:345-352.

Bridge, D.; Göker, M. H.; McGinty, L.; and Smyth, B. 2005. Case-based recommender systems. Knowl. Eng. Rev. 20(3):315-320.

Burke, R. 2002. Hybrid recommender systems: Survey and experiments. User modeling and user-adapted interaction 12(4):331-370.

Campbell, A. T.; Eisenman, S. B.; Lane, N. D.; Miluzzo, E.; Peterson, R. A.; Lu, H.; Zheng, X.; Musolesi, M.; Fodor, K.; and Ahn, G.-S. 2008. The rise of people-centric sensing. IEEE Internet Computing 12(4):12-21.

Cheng, H.-T. 2013. Learning and Recognizing the Hierarchical and Sequential Structure of Human Activities. Ph.D. Dissertation, Carnegie Mellon University, Pittsburgh, PA, USA.

Dearden, P. 2007. Game, set and mismatch. EMBO Reports 8(3):219-219.

Fister, I.; Rauter, S.; Yang, X.-S.; Ljubič, K.; and Fister, I. 2015. Planning the sports training sessions with the bat algorithm. Neurocomput. 149(PB):993-1002.

Glaros, C.; Fotiadis, D. I.; Likas, A.; and Stafylopatis, A. 2003. A wearable intelligent system for monitoring health condition and rehabilitation of running athletes. In 4th International IEEE EMBS Special Topic Conference on Information Technology Applications in Biomedicine, 2003., 276279.

Hermens, H.; op den Akker, H.; Tabak, M.; Wijsman, J.; and Vollenbroek-Hutten, M. 2014. Personalized coaching systems to support healthy behavior in people with chronic conditions. 24(6):815-826.

Lee, I. M., and Skerrett, P. J. 2001. Physical activity and all-cause mortality: what is the dose-response relation? Medicine and science in sports and exercise 33(6 Suppl):S459-71- discussion S493-4.

Liebenberg, L. 2008. The relevance of persistence hunting to human evolution. Journal of human evolution 55(6):1156-1159.
Mattson, M. P. 2012. Evolutionary aspects of human exercise-born to run purposefully. Ageing Research Reviews 11(3):347-352.

Mayer-Schönberger, V., and Cukier, K. 2013. Big data: A revolution that will transform how we live, work, and think. Houghton Mifflin Harcourt.

Möller, A.; Scherr, J.; Roalter, L.; Diewald, S.; Hammerla, N.; Plötz, T.; Olivier, P.; and Kranz, M. 2011. Gymskill: Mobile exercise skill assessment to support personal health and fitness. In 9th Intl. Conf. on Pervasive Computing (Pervasive 2011), Video, San Francisco, CA, USA.

Peek, N.; Combi, C.; Marin, R.; and Bellazzi, R. 2015. Thirty years of artificial intelligence in medicine (aime) conferences: A review of research themes. Artificial Intelligence in Medicine 65(1):61 - 73.

Rauter, S. 2018. New approach for planning the mountain bike training with virtual coach. TRENDS in Sport Sciences (2):69-74.

Ricci, F.; Rokach, L.; and Shapira, B., eds. 2015. Recommender Systems Handbook. Springer.

Rooksby, J.; Rost, M.; Morrison, A.; and Chalmers, M. C. 2014. Personal tracking as lived informatics. In Proceedings of the 32nd annual ACM conference on Human factors in computing systems, 1163-1172. ACM.

Senin, P.; Lin, J.; Wang, X.; Oates, T.; Gandhi, S.; Boedihardjo, A. P.; Chen, C.; and Frankenstein, S. 2018. GrammarViz 3.0: Interactive discovery of variable-length time series patterns. ACM Trans. Knowl. Discov. Data 12(1):10:110:28.

Smyth, B., and Cunningham, P. 2017. Running with cases: A CBR approach to running your best marathon. In CaseBased Reasoning Research and Development - 25th International Conference, ICCBR 2017, Trondheim, Norway, June 26-28, 2017, Proceedings, 360-374.

Smyth, B., and Cunningham, P. 2018a. An analysis of case representations for marathon race prediction and planning. In Case-Based Reasoning Research and Development - 26th International Conference, ICCBR 2018, Stockholm, Sweden, July 9-12, 2018, Proceedings, 369-384.

Smyth, B., and Cunningham, P. 2018b. Marathon race planning: A case-based reasoning approach. In Proceedings of the Twenty-Seventh International Joint Conference on Artificial Intelligence, IJCAI 2018, July 13-19, 2018, Stockholm, Sweden., 5364-5368.

Smyth, B. 2007. Case-based recommendation. In The Adaptive Web, Methods and Strategies of Web Personalization, 342-376.

Smyth, B. 2018. Fast starters and slow finishers: A largescale data analysis of pacing at the beginning and end of the marathon for recreational runners. Journal of Sports Analytics 4(3):229-242.

Yoganathan, D., and Kajanan, S. 2013. Persuasive technology for smartphone fitness apps. In 17th Pacific Asia Conference on Information Systems, PACIS 2013, Jeju Island, Korea, June 18-22, 2013, 185. 